\title{
Anthocyanin Improving Metabolic Disorders in Obese Mice from Aornia melanocarpa
}

\author{
Jie Wei ${ }^{1 *}$, Guokun Zhang ${ }^{1}$, Xiao Zhang', Jun gao', Jungang Fan ${ }^{2}$, Zhiquan Zhou ${ }^{2}$ \\ ${ }^{1}$ School of Life Science, Liaoning University, Shenyang 110036, CHINA. \\ ${ }^{2}$ Foresty Science Research Institute of Liaoning Province, Shenyang, CHINA.
}

\begin{abstract}
The aim of this study was to investigate the effects of Aornia melanocarpa anthocyanin (AMA) on symptoms of metabolic syndrome in obese mouse. AMA can reduce weight. Blood glucose and lipid metabolism were significantly improved, and so as hepatic lipid metabolism. This suggesting AMA is a strong indication of improved glucoselipid metabolism and weight. Moreover, both morphologic and histological detections indicated that AMA significantly reversed obsity induced hepatic steatosis and liver injury. Furthermore, AMA significantly ameliorated inflammatory cytokines including both IL- 6 and TNF- $\alpha$ in liver and fatty tissue. Moreover, insulin receptor (IR), p-IRS and p-AKT that associated proteins about glucose-lipid metabolism signaling pathway were markedly up-regulated at protein levels by AMA. Our results demonstrate that Aornia melanocarpa anthocyanin treatment can alleviate obsity and metabolic syndrome. The beneficial effects of AMA are associated with improved glucose-lipid metabolism and reduced levels of inflammatory cytokines.
\end{abstract}

Key words: Metabolic disorders, Obese, Aornia melanocarpa, Anthocyanin, Mechnism.

\section{INTRODUCTION}

The obesity epidemic is a global issue and shows no signs of abating. ${ }^{1,2}$ while the cause of this epidemic remains unclear. ${ }^{3,4}$ And Obesity is the main cause of metabolic disorders. ${ }^{5}$ Marketing practices of energydense foods and institutionally-driven declines in physical activity are the alleged perpetrators for the epidemic, despite a lack of solid evidence to demonstrate their causal role..$^{6-10}$ Considering the role of such putative etiological factors of obesity may lead to comprehensive, cause specific, and effective strategies for prevention and treatment of this global epidemic. Fruits and vegetables have abundance and variety of bioactive compounds present. As an alternative to pharmaceutical medications, consumption of diets rich in natural bioactive components and their contribution to improving metabolic disorders and obese has been a subject of considerable interest to researchers. Many published cohort studies suggest that high intakes of flavonoids may be associated with a decreased risk of metabolic disorders. ${ }^{11-14}$

Aronia Melanocarpa, also called black chokeberry, is a member of the Rosaceae family. Shrubs of the aronia genus are native North American plants that have been traditionally used in Native American medicine, for example in the treatment of colds ${ }^{15,16}$ The aronia plant has become popular in recent years due to its berries having a high content of anthocyanins with antioxidant activity. Aronia products are well received as nutritional supplements, and the berries are also used as an ingredient for juices, wines, jams, and as a source of natural food colorants. ${ }^{16}$ In recent years, black chokeberries have gained popularity due to their high content of polyphenols with antioxidant activity. In fact, they possess the highest anti oxidant activity among berries and other fruits investigated so far as measured with the oxygen
Submission Date : 24-12-2015 Revision Date : 29-02-2016 Accepted Date : :29-02-2016

DOI: 10.5530/ijper.50.3.8 Correspondence: Jie Wei

Chongshan Middle road 66, Huanggu District, School of Life Science of Liaoning University, Shenyang, CHINA. Zip code: 110036

Tel: $+86-24-62202232$

Fax:+86-24-62202074

E-mail:weijie@Inu.edu.cn

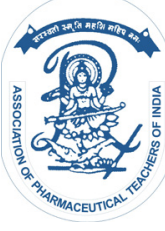

www.ijper.org 
radical scavenging capacity (ORAC) assay. ${ }^{16,17}$ The Aronia berries contain high levels of flavonoids, mostly anthocyanins and proanthocyanidins. The formation of free radicals is strongly associated with lipid peroxidation and has also been implicated in the development of a variety of diseases, including cellular aging, mutagenesis, inflammation, carcinogenesis, coronary heart disease and diabetes. Anthocyanins are a group of abundant and widely consumed flavonoid constituents possessing a characteristic C3-C6-C3 carbon structure that exists in many fruit- and vegetable-based food products. Their intake has been estimated to be up to 9-fold higher than that of other dietary flavonoids.

Our preliminary work has focused on identification of Aornia melanocarpa anthocyanin (AMA) and the antioxidant properties, which included anti-lipid peroxidation capability, and superoxide anion radical-scavenging activity, hydroxyl radical-scavenging activity. The results of the study were similar to those of other scholars. ${ }^{18,19}$ We detected AMA containing cyanidin 3-galactoside, cyanidin 3-glucoside, cyanidin 3-arabinoside and cyanidin 3 -xyloside. To continue the previous study, the current study was aimed at demonstrating the therapeutic effect of anthocyanins from Aronia fruits against metabolic disorders in obese mouse model, and further addressing its potential mechanisms. The results suggested that AMA could offer a promising source of agent that may have the potential to be used as food health products.

\section{MATERIALS AND METHODS}

Reagents and diets: Aronia Melanocarpa fruit was provided by Liaoning Academy of Forestry (Shenyang, China), and the Aronia fruits polyphones was extract by Liaoning University (Shenyang, China). All the chemicals used were of analytical grade. The high-fat diet (HFD; $50 \%$ energy as fat, $20 \%$ protein, $30 \%$ carbohydrate) and the normal-fat diet (NFD; $10 \%$ fat, $20 \%$ protein, $70 \%$ carbohydrate).

Animal care and experimental protocol: All protocols in this study were performed in accordance with the National Institutes of Health regulations for the care and use of animals in research. Five weeks KM mice (23-27 g, Benxi Changsheng Laboratory Animal Technology Co., Ltd) were maintained at a 12:12 h light: dark cycle, and given water ad libitum. Animals were housed in cages individually in an environmentally controlled room at $21 \pm 2.0^{\circ} \mathrm{C}$ and $50 \% \pm 5 \%$ humidity.

Establishment of mice obesitymodel and anthocyanins supplementation: After one week of adaptation, female mice (6 weeks of age) were randomly divided into a normal-fat diet (NFD) group $(n=10)$ and
4 high-fat diet (HFD) groups $(\mathrm{n}=10 \times 3)$ by weight and fed for 8 weeks, respectively. During this period, 3 HFD groups were fed with a HFD, a HFD+150 $\mathrm{mg} \cdot \mathrm{L}^{-1}$ and $300 \mathrm{mg} \cdot \mathrm{L}^{-1} \mathrm{AMA}$, respectively. Mice in the control group $(\mathrm{n}=8)$ were maintained with NFD. Blood glucose, body weight and food intake were measured weekly, respectively. Sample collection: After the treatment for 8 weeks,mice were sacrificed by $\mathrm{CO}_{2}$ inhalation followed by cervical dislocation, and blood was collected from the eyes. Serum was obtained and stored at $-80^{\circ} \mathrm{C}$ until analysis. Tissues, including liver, kidney, spleen, visceral fat were removed, weighted and snap-frozen in liquid nitrogen, and stored immediately at $-80^{\circ} \mathrm{C}$.

Lipid, adiponectin and insulin in serum were measured: The blood serum of mice received centrifugation to measure triglyceride (TG), total cholesterol (TC), low density lipoprotein cholesterol (LDL-C), high density lipoprotein cholesterol (HDL-C), adiponectin $(\mathrm{ADPN})$ and insulin levels were measured using Kit (Nanjing Jiancheng Bioengineering Institute, China). For liver lipid measurement, we followed the manufacturer's instruction.

Liver TG,TC and lipid measurement: To analyze hepatic lipid levels, liver tissue $(50 \mathrm{mg}$ ) was homogenized in $1 \mathrm{ml} \mathrm{80 \%} \mathrm{isopropyl} \mathrm{alcohol.} \mathrm{After} \mathrm{centrifugation} \mathrm{at}$ $5000 \mathrm{~g} \mathrm{~min}^{-1}$ for $10 \mathrm{~min}$, the supernatant was collected, and then TG, TC and lipid levels were measured using Kit (Nanjing Jiancheng Bioengineering Institute, China). For liver lipid measurement, we followed the manufacturer's instruction.

Histological examination: At the time of killing, small pieces of liver were fixed immediately in 10\% buffered formalin. As previously reported, 21 after paraffin embedding, $5 \mathrm{~mm}$ sections were deparaffinized in xylene and were rehydrated through a series of decreasing concentrations of ethanol. Sections were stained with hematoxylin and eosin (HE). Photomicrographs were taken on an Olympus BX 51 microscope (OLYMPUS Co. Ltd, Tokyo, Japan).

RNA extraction and quantitative RT-PCR: Liver tissues $(20 \mathrm{mg})$ and visceral fat $(20 \mathrm{mg})$ were ground with the liquid nitrogen in RNase-free mortars, respectively. Total RNA was extracted from three individual mice per strain per diet group. The quality and quantity of RNA was determined. Quantitative real-time PCR analysis was performed using the ABI Prism 7900-HT sequence detection system ( 96 wells) as described.22 The following primers were designed for mouse cytokine analysis and were synthesized: tumor necrosis factor- $\alpha(\mathrm{TNF}-\alpha)$, forward 5'-CССTCACACTCAGATCATCTTCT-3' and reverse 5'-GCTACGACGTGGGCTACAG-3'; IL-6， forward 
5'-TAGTCCT'TCCTACCCCAATTTCC-3' and reverse 5'-TTGGTCCTTAGCCACTCCTTC-3'.

Western blotting analyses: Extract the protein from each of the liver tissues. In brief, 22 the ground powder of frozen tissues was placed in RIPA cell lysis buffer (Cell Signaling Technology, USA) for $15 \mathrm{~min}$ on ice. After brief sonication, the cell lysate was centrifuged at $13500 \mathrm{rpm}$ for 15 minutes at $4^{\circ} \mathrm{C}$. The supernatant was fractionated by electrophoresis in a 12\% SDS-polyacrylamide gel and transferred to a nitrocellulose membrane. The following primary antibodies were used for immunoblotting: anti-IR, anti-p-IR, anti-p-AKT and antitubulin (Bio Basic Inc., Canada). Signals were detected by an enhanced chemiluminescence technique (Amersham Life Science, USA).

Statistical analyses: One-way analysis of variance (ANOVA), followed by the post hoc test, was performed to analyze the data with SPSS software (SPSS16; Beijing Stats Data Mining, Beijing, China). Data were expressed as mean \pm S.E.M., and $\mathrm{P}<0.05$ was considered statistically significant.

\section{RESULTS}

\section{Anthocyanins reducethe body weight and calorie intakeof HFD-induced obesity}

All the anthocyanins treatment group mice are with glossy hair and a sensitive reflection ability and food ration. Dynamic change of body weight was measured during the 8 week feeding period. As the data shows in Figure 1A, body weight gradually increased over time of the HFD fed period, and the AMA groups showed significant reduction compared to HFD group in the 6-8 weeks. Figure 1B showed that the calorie intake (kcal/day) of all the groups. The calorie intake of obsity mice in HFD group were higher than NFD group significantly $(\mathrm{P}<0.05)$. Fortunately, the treatment of $300 \mathrm{mg} \cdot \mathrm{L}^{-1}$ anthocyanins could reduce the calorie intake significantly $(\mathrm{P}<0.05)$. This shows that obesity might occur during this period because of the excessive caloric intake.

\section{Anthocyanins improvethe organ hypertrophy and visceral fat excessof HFD-induced obesity}

As the Figure 2A showed that 8 weeks of HFD feeding resulted in liver enlargement of mice significantly $(\mathrm{P}<0.01)$, and the treatment of $300 \mathrm{mg} \cdot \mathrm{L}^{-1} \mathrm{AMA}$ could improve the liver enlargement significantly $(\mathrm{P}<0.05)$. In addition, there was no obvious change in the liver and kidney during this period. As the Figure 2B showed the changes of visceral fat and liver index were similar. The visceral fat of obese mice HFD-induced was more than normal mice significantly $(\mathrm{P}<0.001)$, and the treatment of $300 \mathrm{mg} \cdot \mathrm{L}^{-1}$ AMA could restore the visceral fat to normal. This suggested that the metabolic disorders and visceral fat in mice may be caused by liver injury, and the liver injury is repaired by anthocyanins to reverse the disease.

\section{Anthocyanins regulate the blood lipids and glucose of HFD-induced obesity}

The fasting-state plasma triglycerides (TG), total cholesterol (TC), low density lipoprotein cholesterol (LDL-C) and high density lipoprotein cholesterol (HDL-C) levels of mice are shown in Figure 3A. HFD mice had abnormal serum lipid levels, which may be caused by the less efficient use of fat from the diet or obesty. The TG, TC and LDL-C levels of AMA treatment mice were lower significantly $(\mathrm{P}<0.001$ or $\mathrm{P}<0.01)$ than the mice of HFD group. This may suggest that AMA improve the lipid metabolism. The HDL-C level increased significantly with increasing AMA content $(\mathrm{P}<0.05, \mathrm{P}<0.001)$. The blood glucose level was reported in Figure 3B. As shown, the NFD group was in the flat below 10 $\mathrm{mmol} \cdot \mathrm{L}^{-1}$, and HFD group increased rapidly in these weeks. In the $4^{\text {th }}$ week, anthocyanins groups were lower than HFD group significantlt $(\mathrm{P}<0.01)$. And in the $8^{\text {th }}$ week effect to achieve the best.

\section{Anthocyanins regulate the blood insulin and ADPN of HFD-induced obesity}

The fasting-state plasma insulin and adiponectin (ADPN) levels of mice were shown in Figure 4A, B. The insulin level of anthocyanins treatment was lower than HFD group $(\mathrm{P}<0.001)$. And AMA can induce the ADPN level $(\mathrm{P}<0.05, \mathrm{P}<0.01)$, and the level of 300 $\mathrm{mg} \cdot \mathrm{L}^{-1}$ treatment was higher than NFD. This suggested that AMA regulates their level to reduce blood glucose and lipids.

\section{Anthocyanins regulate the lipid levels in liver of HFD-induced obesity}

At the end of the experiment period ( 8 weeks), the hepatic lipids, TG and TC of mice were determined, as shown in Figure 5. AMA treatment reduced the lipids level in liver significantly compared to HFD group $\left(150 \mathrm{mg} \cdot \mathrm{L}^{-1} \mathrm{P}<0.01,300 \mathrm{mg} \cdot \mathrm{L}^{-1} \mathrm{P}<0.001\right)$. The treatment of $300 \mathrm{mg} \mathrm{L}^{-1} \mathrm{AMA}$ have obvious effect on $\mathrm{TG}(\mathrm{P}<0.05)$ and TC $(\mathrm{P}<0.01)$. These results suggested that the AMA treatment might decrease the lipid production and storage in liver. This may suggest that AMA improve the lipid metabolism of hepatocyte. 


\section{Anthocyanins ameliorate HFD-induced hepatic and adipose tissue inflammation}

To further investigate the effect of AMA treatment on hepatic injury and metabolic disorders in HFD mice, the levels of inflammatory markers, IL- 6 and TNF- $\alpha$ were measured in liver and fatty tissue. As the results show in Figure 6A, in the liver tissue, the levels of inflammatory markers (IL- 6 and TNF- $\alpha$ ) of NFD group mice were lower than HFD group mice significantly $(\mathrm{P}<0.001$, $\mathrm{P}<0.05)$, and AMA can reduce the levels significantly $(\mathrm{P}<0.01)$. As the Figure 6B, the levels of inflammatory markers in fatty tissue was similar to liver tissue, and the effect was more obviously $(\mathrm{P}<0.01$ or $\mathrm{P}<0.001)$. The data suggested that AMA treatment significantly decreased the elevation in hepatic and adipose inflammatory markers.

\section{Anthocyanins reverse HFD-induced liver injury}

After 8 weeks, AMA treatment, the pathological alterations of livers from different groups were firstly evaluatedby morphology and histological (HE staining) examination (Figure 7). Under microscopy, for NFD group, the structure of liver tissue is arranged neatly, the size of central venous is normal, and the sinuses are seen in the middle of the central venous. Moreover, the morphology of hepatocyte is clear, the membrane is intact and the nucleolus is clearly visible. The HFD group significantly induced massive hepatic steatosis and inflammatory cells infiltration, arranged hepatocytes into hierarchical disorder, swelled the cells, increased the size of the nucleus, decreased the consolidation of the boundaries of the hepatocytes, and eliminated some parts of the nucleolus. The anthocyanins groups supplementation obviously reversed the abovementioned HFD induced adverse changes.

\section{Anthocyanins up-regulate the expression of IR, p-IR and $\mathrm{p}-\mathrm{AKT}$}

As shown in Figure 8, the protein (IR, p-IR and p-Akt) expression decreased significantly in the HFD group compared to NFD group. The HFD induced the expression decrease of IR, p-IR and p-Akt in liver, but AMA could increase significantly them. The pharmacological study showed that HFD induced insulin resistanceand anthocyanins could improve the insulin sensitivity because it can increase expression of IR, $\mathrm{p}-\mathrm{IR}$ and $\mathrm{p}-\mathrm{AKT}$.

\section{DISCUSSION}

Our study was carried out to study the efficacy of Aornia melanocarpa fruit anthocyanins as treatment for metabolic disorders in obsity. Our results demonstrate that supplementation with anthocyanins significantly decreases body weight, liver index, calorie intake and visceral fat. Blood index was also improved that serum TG, TC, LDL-C, insulin and glucose were decreased significantly by anthocyanins, as well the serum ADPN and HDL-C was increased significantly. Furthermore anthocyanins significantly improves metabolic disorders, involving hepatic steatosis, inflammatory cytokines, and liver injury in mice fed with a HFD diet. Histological and biochemical analyses show that diet supplementation with anthocyanins reduced the development of hepatic steatosis and injury induced by the HFD diet. The decrease of inflammatory cells infiltration determined by histopathological analysis, and the reduction of hepatic lipids levels demonstrate that supplementation with anthocyanins reduced the fat accumulation in the liver. Anthocyanins significantly alleviated adipose and hepatic inflammation, evidenced by the reduction of HFD-induced increase of IL- 6 and TNF- $\alpha$ mRNA expressions in liver. Activation of the insulin receptor (IR) triggers its intrinsic protein-tyrosine (Tyr) kinase activity, resulting in autophosphorylation of several IR Tyr residues and the recruitment and phosphorylation of IR substrate (IRS) proteins. ${ }^{20}$ After Tyr phosphorylation, IRS acts as docking protein for several Src homology 2 (SH2) domain-containing molecules, including Grb2, the small adapter protein Nck, the tyrosine phosphatase Syp, phosphatidylinositol 3-kinase (PI3K), and others. ${ }^{21}$ PI3K has a central role downstream of the IRS proteins in the activation and regulation of many insulin-induced metabolic processes. During its association with IRS, PI3K promotes the activation of Akt (PKB) that regulates multiple biological processes. The duration and extent of signals induced by insulin are tightly regulated to promote optimal insulin actions in the body, and impaired generation of these signals causes a common pathological state termed insulin resistance. This occurs in a wide variety of pathological states, including obesity, hypertension, chronic infection and cardiovascular diseases ${ }^{22-24}$ The expression of protein IR, p-IR and p-AKT was also increased with anthocyanins, this suggested that anthocyanins can promote the operation of the insulin signaling pathway, increase insulin sensitivity, and then promote the absorption of glucose to achieve the effect of weight loss. ${ }^{20,24-26}$ And the treatment of anthocyanins $300 \mathrm{mg} \cdot \mathrm{L}^{-1}$ had the best effect in all these groups. To the best of our knowledge, this study is the first to show the therapeutic role of anthocyanins in a HFDinduced obsity mouse model. anthocyanins are generally considered to be safe and have minimal adverse effects. Neither mortality nor any signs of toxicity with orally administrating a single dose (as high as $2000 \mathrm{mg} \mathrm{kg}^{-1}$ ) 


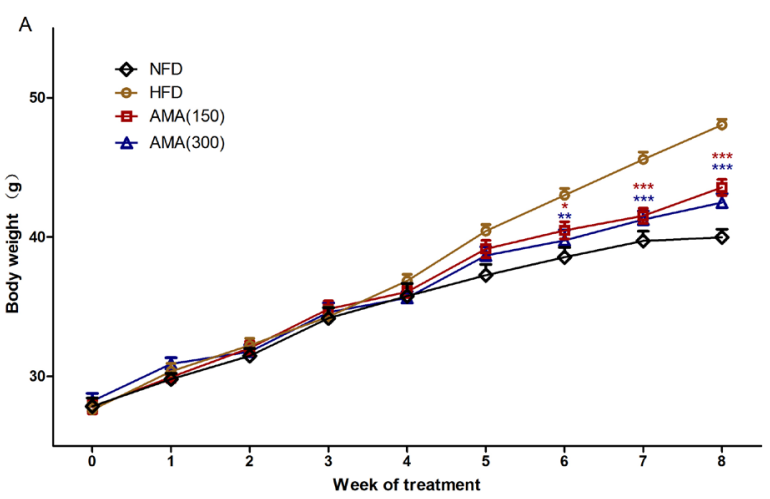

Figure 1 (a): Effects of AMA on the body weight (A) and calorie intake $(B)$ of all the experimental mice.

Values are expressed as mean \pm SEM. ${ }^{*} p<0.05$ vs. HFD group. ${ }^{* \star} p<0.01$ vs. HFD group. ${ }^{* *} P<0.001$ vs. HFD group.

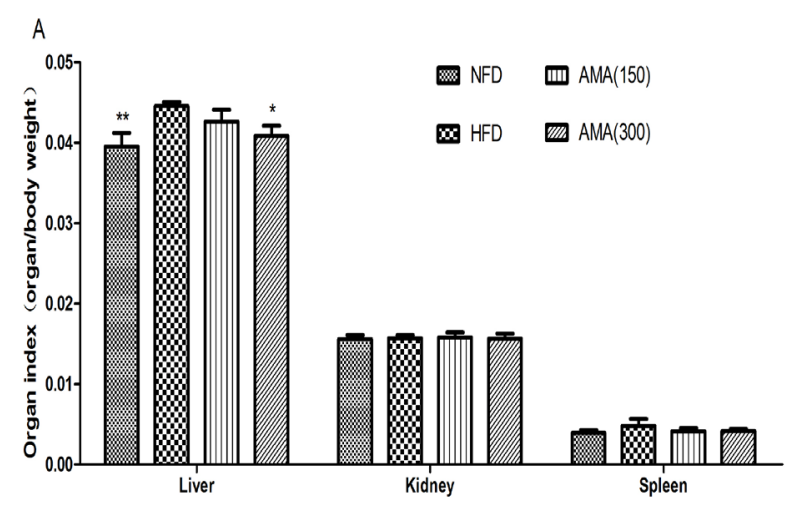

Figure 2 (a): Effects of AMA on the organ index (A) and visceral fat $(B)$ of all the experimental mice.

Values are expressed as mean \pm SEM. ${ }^{*} p<0.05$ vs. HFD group. ${ }^{* *} p<0.01$ vs. HFD group. ${ }^{* \star *} P<0.001$ vs. HFD group.

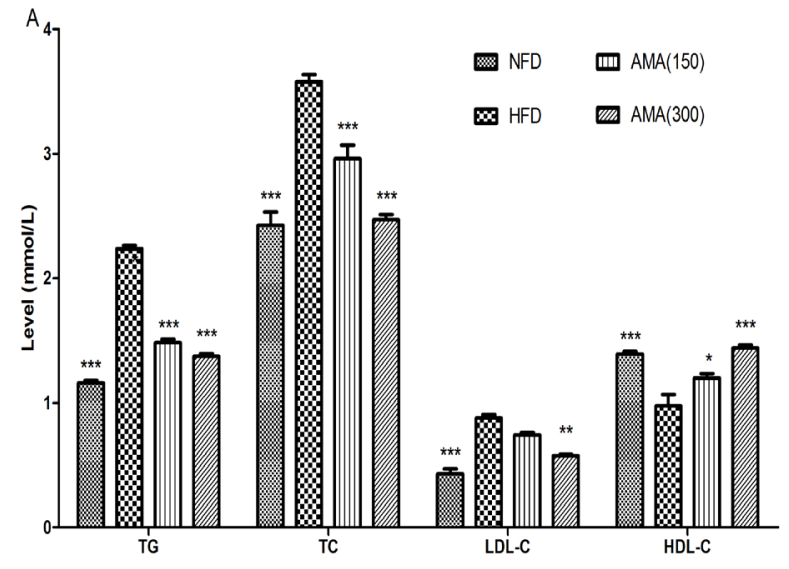

Figure 3 (a): Effects of AMA on the blood lipids (A) and blood glucose $(B)$ of all the experimental mice.

Values are expressed as mean \pm SEM. ${ }^{*} p<0.05$ vs. HFD group. ${ }^{* *} p<0.01$ vs. HFD group. ${ }^{\star * \star} \mathrm{P}<0.001$ vs. HFD group.

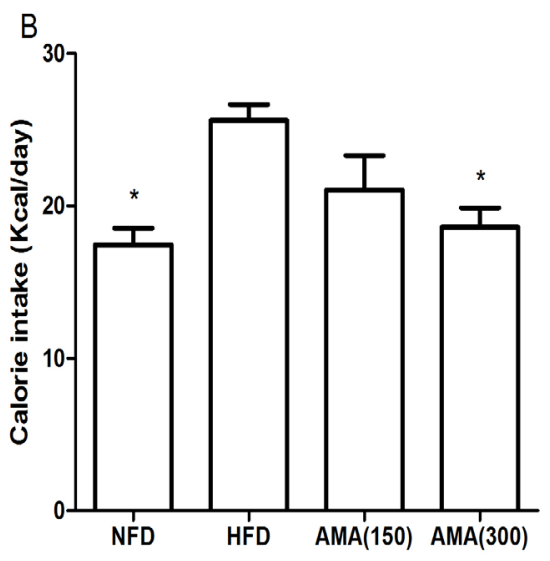

Figure 1 (b): Effects of AMA on the body weight (A) and calorie intake (B) of all the experimental mice.

Values are expressed as mean \pm SEM. ${ }^{*} p<0.05$ vs. HFD group. ${ }^{* \star} p<0.01$ vs. HFD group. ${ }^{* * *} \mathrm{P}<0.001$ vs. HFD group.

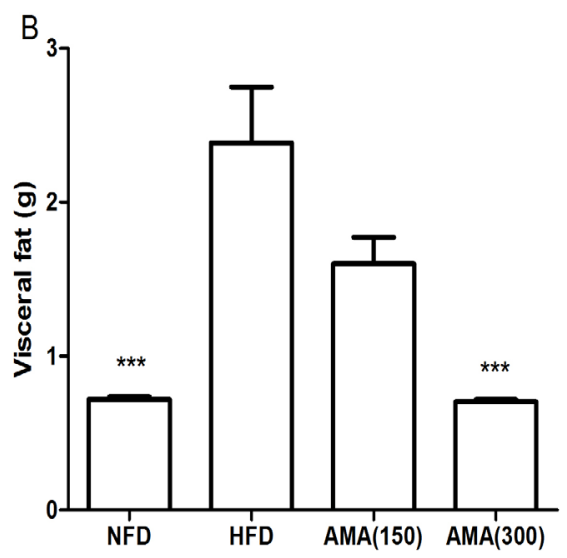

Figure-2 (b): Effects of AMA on the organ index (A) and visceral fat $(B)$ of all the experimental mice.

Values are expressed as mean \pm SEM. ${ }^{*} p<0.05$ vs. HFD group. ${ }^{* *} p<0.01$ vs. HFD group. ${ }^{* * *} \mathrm{P}<0.001$ vs. HFD group.

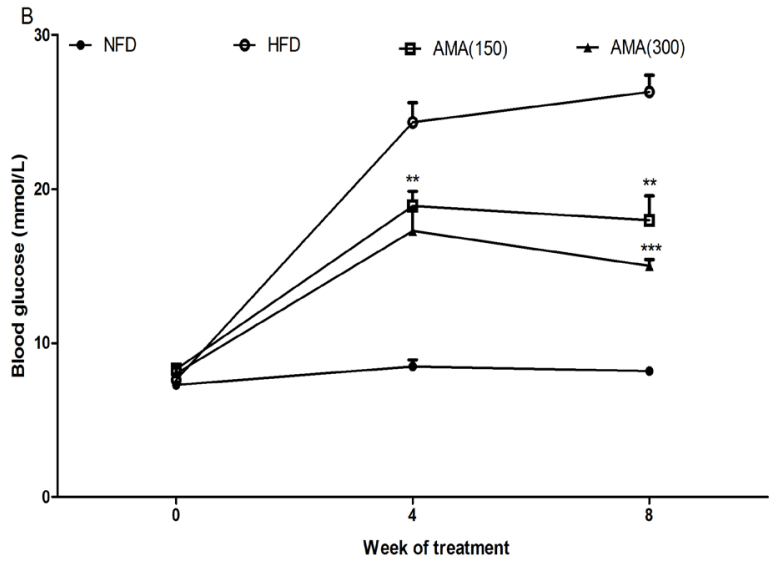

Figure 3 (b): Effects of AMA on the blood lipids (A) and blood glucose $(B)$ of all the experimental mice.

Values are expressed as mean \pm SEM. ${ }^{*} p<0.05$ vs. HFD group. ${ }^{* *} p<0.01$ vs. HFD group. ${ }^{* \star *} P<0.001$ vs. HFD group. 


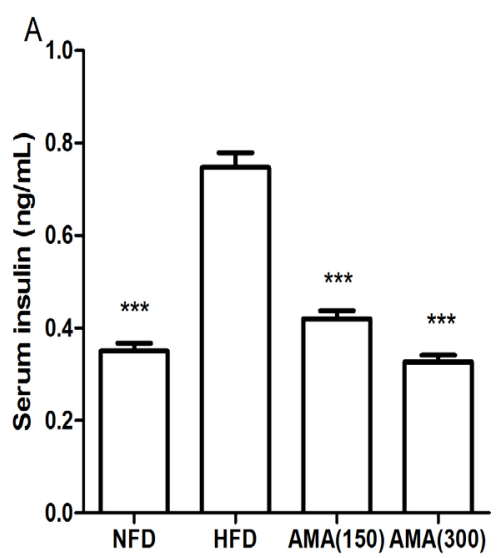

Figure 4 (a): Effects of AMA on the blood insulin (A) and blood ADPN (B) of all the experimental mice.

Values are expressed as mean \pm SEM. ${ }^{*} p<0.05$ vs. HFD group. ${ }^{* *} p<0.01$ vs. HFD group. ${ }^{\star \star \star} P<0.001$ vs. HFD group.

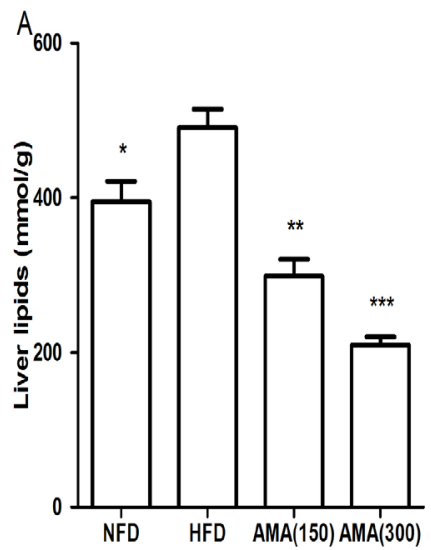

Figure 5 (a): Effects of AMA on lipid levels in liver of all the experimental mice. lipids (A), TG (B) and TC (C).

Values are expressed as mean \pm SEM. ${ }^{*} p<0.05$ vs. HFD group. ${ }^{* *} P<0.01$ vs. HFD group. ${ }^{* \star *} \mathrm{P}<0.001$ vs. HGFD group.

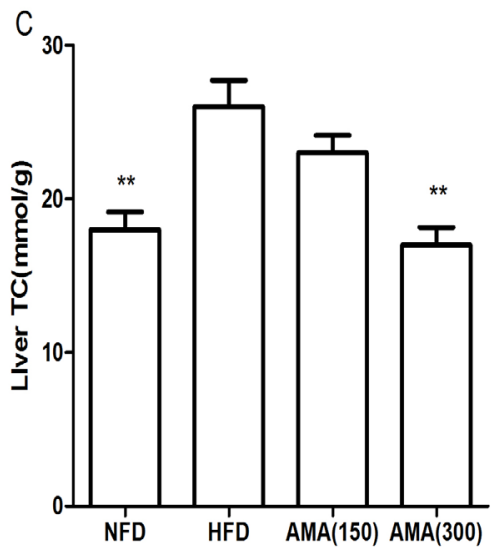

Figure 5 (c): Effects of AMA on lipid levels in liver of all the experimental mice. lipids (A), TG (B) and TC (C).

Values are expressed as mean \pm SEM. ${ }^{*} p<0.05$ vs. HFD group. ${ }^{* *} \mathrm{P}<0.01$ vs. HFD group. ${ }^{* * *} \mathrm{P}<0.001$ vs. HGFD group.

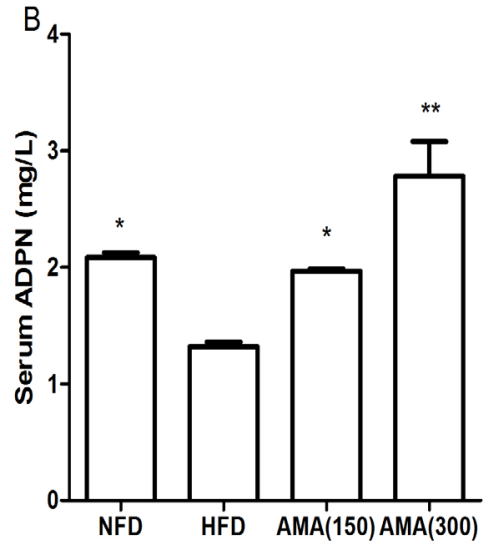

Figure 4 (b): Effects of AMA on the blood insulin (A) and blood ADPN (B) of all the experimental mice.

Values are expressed as mean \pm SEM. ${ }^{*} p<0.05$ vs. HFD group. ${ }^{* *} p<0.01$ vs. HFD group. ${ }^{* * *} \mathrm{P}<0.001$ vs. HFD group.

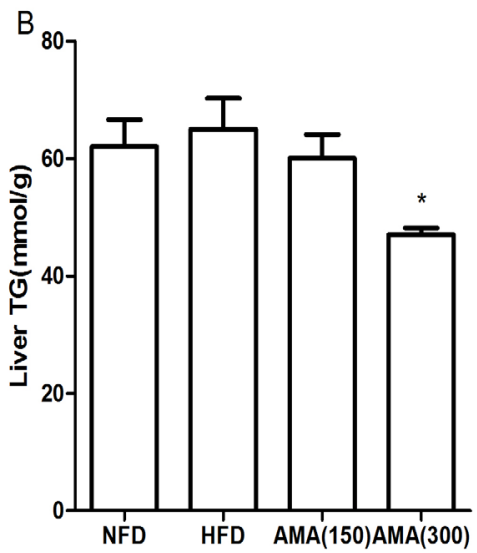

Figure 5 (b): Effects of AMA on lipid levels in liver of all the experimental mice. lipids (A), TG (B) and TC (C).

Values are expressed as mean \pm SEM. ${ }^{*} \mathrm{p}<0.05$ vs. HFD group. ${ }^{* *} \mathrm{P}<0.01$ vs. HFD group. ${ }^{* \star *} \mathrm{P}<0.001$ vs. HGFD group.

was observed in the acute toxicity study in mice in our preliminary study (Data are not shown). These results suggest that the beneficial effects of anthocyanins may be mediated by improved glucose-lipid metabolism. The possible beneficial effects of anthocyanins in obsity and diabetes mellitus need to be further investigated.

In conclusion, drinking fluid supplementation with anthocyanins significantly reduces the development of obesity, insulin resistance, hypercholesterolemia and hepatic steatosis in mice fed the HFD diet, which mimics many syndromes of metabolic disorders. These results suggest that the beneficial effects of anthocyanins may be mediated by improved lipid metabolism and decreased levels of inflammatory cytokines such as TNF-á and IL-6. The possible beneficial effects of anthocyanins in diabetic or pre-diabetic patients need to be further investigated. 


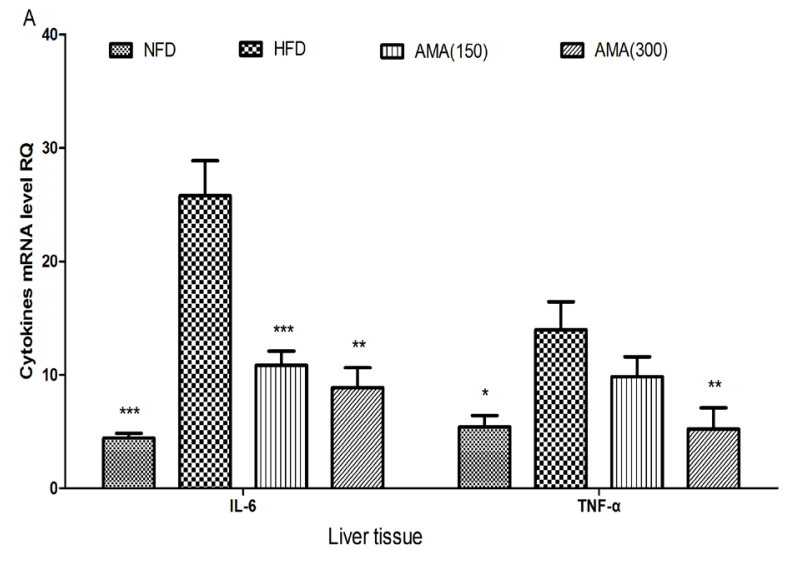

Figure 6 (a): Effects of AMA on inflammatory cytokines release of all the experimental mice. Relative mRNA expression of IL- 6 and TNF-amRNAs was measured by quantitative real-time PCR ( $n \geq 3$ per group). Liver tissue

(A) and fatty tissue (B)

Values are expressed as mean \pm SEM. ${ }^{*} p<0.05$ vs. HFD group. ${ }^{* *} p<0.01$ vs. HFD group. ${ }^{* * *} \mathrm{P}<0.001$ vs. HFD group.

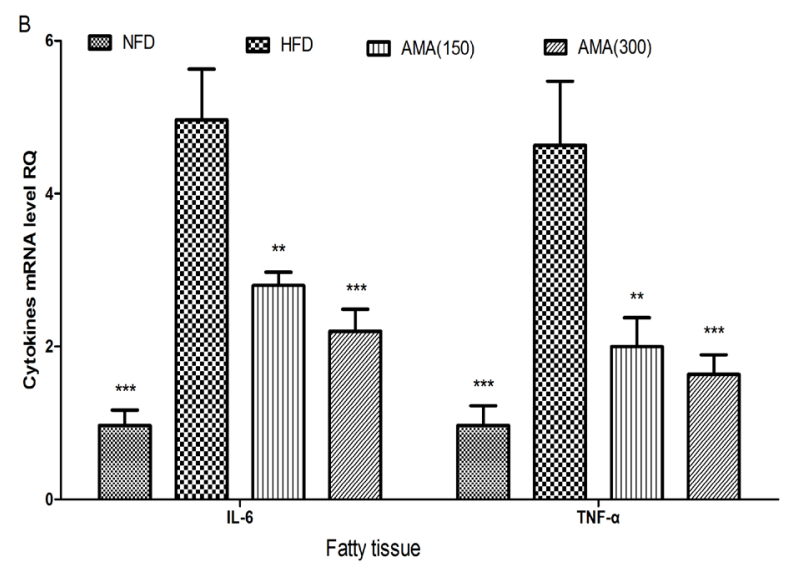

Figure 6 (b); Effects of AMA on inflammatory cytokines release of all the experimental mice. Relative mRNA expression of IL- 6 and TNF-amRNAs was measured by quantitative real-time PCR ( $n \geq 3$ per group). Liver tissue (A) and fatty tissue (B).

Values are expressed as mean \pm SEM. ${ }^{*} p<0.05$ vs. HFD group. ${ }^{* *} p<0.01$ vs. HFD group. ${ }^{* * *} \mathrm{P}<0.001$ vs. HFD group.

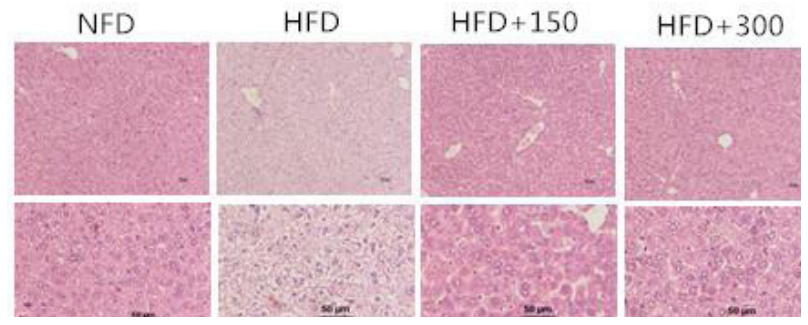

Figure 7: Effects of AMA on liver pathology in experimental mice.

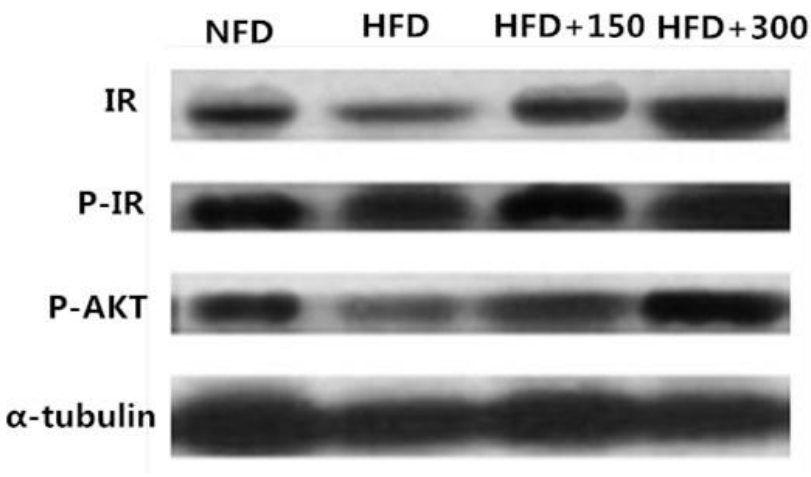

Figure 8: Effects AMA on the alterations of proteins associated with liver glucose and lipid metabolism in experimental mice. The expressions of IR, $p$-IR and $p$-AKT protein levels were measured by western blot. Each group contains at least 4 mice, and the representative band was shown. Values are expressed as mean \pm SEM. ${ }^{*} p<0.05$ vs. HFD group. ${ }^{* *} P<0.01$ vs. HFD group. ${ }^{* \star} P<0.001$ vs. HFD group.

\section{ACKNOWLEDGEMENTS}

This work was supported by grants from Science and Technology Government Department Fund in Liaoning province (Grant No. L2011008; UPRP20140428), the Foundation of Ocean and fishery hall in Liaoning province (Grant No.201407), the Social Science fund plan in Liaoning province (Grant No. L15BJY029) and China National Natural Science Foundation of China.

\section{CONFLICT OF INTEREST}

There is no potential conflict of interest to declare.

\section{REFERENCES}

1. Davis B, and Wansink B. Fifty years of fat: news coverage of trends that predate obesity prevalence. BMC Public Health. 2015;15(1):629.

2. Ogden $\mathrm{CL}$, Carroll MD and Flegal KM. High body mass index for age among US children and adolescents, 2003-2006. JAMA. 2008;299(20);2401-5.

3. Bray GA and Champagne CM. Beyond energy balance: there is more to obesity than kilocalories. J Am Diet Assoc. 2005;105(5 Suppl 1):S17-23.

4. Eisenmann JC. Insight into the causes of the recent secular trend in pediatric obesity: Common sense does not always prevail for complex, multi-factorial phenol types. Prev Med. 2006;42(5):329-35

5. Samocha-Bonet D, Dixit VD, Kahn CR, Leibel RL, Lin X et al. Metabolically healthy and unhealthy obese--the 2013 Stock Conference report. Obes Rev. 2014;15(9):697-708.

6. Hall KD, Guo J, Dore M and Chow CC. The progressive increase of food waste in America and its environmental impact. PLoS One. 2009;4(11):e7940.

7. McCrory MA, Suen VM and Roberts SB. Biobehavioral influences on energy intake and adult weight gain. J Nutr. 2002;132(12):3830S-4S.

8. Oliver JE. The politics of pathology: how obesity became an epidemic disease. Perspect Biol Med. 2006;49(4):611-27.

9. Prentice $A$ and Jebb S. Energy intake/physical activity interactions in the homeostasis of body weight regulation. Nutr Rev. 2004;62(7 Pt 2):S98-104.

10. Swin burn BA, Sacks G, Hall KD, McPherson K, Fine good DT, Moodie ML, and Gortmaker SL. The global obesity pandemic: shaped by global drivers and local environments. Lancet.2011;378(9793):804-14.

11. Grace MH, Ribnicky DM, Kuhn P, Poulev A, Logendra S, Yousef GG, Raskin I, and Lila MA. Hypoglycemic activity of a novel anthocyanin-rich formulation 
from lowbush blueberry, Vaccinium angustifolium Aiton. Phytomedicine. 2009;16(5):406-15.

12. Guo H, Li D, Ling W, Feng X, and Xia M. Anthocyanin inhibits high glucoseinduced hepatic mtGPAT1 activation and prevents fatty acid synthesis through PKCzeta. J Lipid Res. 2011;52(5):908-22.

13. Kumar S and Pandey AK. Chemistry and biological activities of flavonoids: an overview. Scientific World Journal. 2013;2013:162750.

14. Valenti L, Riso P, Mazzocchi A, Porrini M, Fargion S, and Agostoni C. Dietary anthocyanins as nutritional therapy for nonalcoholic fatty liver disease. Oxid Med Cell Longev. 2013;2013:145421.

15. Kokotkiewicz A, Jaremicz Z, and Luczkiewicz, M. Aronia plants: a review of traditional use, biological activities, and perspectives for modern medicine. J Med Food. 2010;13(2):255-69.

16. Kulling SE, and Rawel, HM. Chokeberry (Aronia Melanocarpa) - A review on the characteristic components and potential health effects. Planta Med. 2008;74(13):1625-34.

17. Zheng $\mathrm{W}$ and Wang, SY. Oxygen radical absorbing capacity of phenolics in blueberries, cranberries, chokeberries, and lingonberries. J Agric Food Chem. 2003;51(2):502-9.

18. Olszewska M. Separation of quercetin, sexangularetin, kaempferol and isorhamnetin for simultaneous HPLC determination of flavonoid aglycones in inflorescences, leaves and fruits of three Sorbus species. J Pharm Biomed Anal. 2008;48(3):629-35.
19. Olszewska MA and Michel P. Antioxidant activity of inflorescences, leaves and fruits of three Sorbus species in relation to their polyphenolic composition. Nat Prod Res. 2009;23(16):1507-21.

20. Saltiel AR, and Kahn, CR. Insulin signalling and the regulation of glucose and lipid metabolism. Nature. 2001;414(6865):799-806.

21. Combettes-Souverain M, and Issad T. Molecular basis of insulin action. Diabetes Metab. 1998;24(6):477-89.

22. Muniyappa R, Montagnani M, Koh KK and Quon MJ. Cardiovascular actions of insulin. Endocr Rev. 2007;28(5):463-91.

23. Reaven G, Abbasi F, and McLaughlin T. Obesity, insulin resistance, and cardiovascular disease. Recent Prog Horm Res. 2004;59:207-23.

24. Riccardi G, Giacco R, and Rivellese AA. Dietary fat, insulin sensitivity and the metabolic syndrome. Clin Nutr. 2004;23(4):447-56.

25. Jimenez-Gomez Y, Cruz-Teno C, Rangel-Zuniga OA, Peinado JR, and Lopez-Miranda J. et al. Effect of dietary fat modification on subcutaneous white adipose tissue insulin sensitivity in patients with metabolic syndrome. Mol Nutr Food Res. 2014;58(11):2177-88.

26. Li C, and Zhang BB. Insulin signaling and action: glucose, lipids, protein. In L. J. (Eds.), Endotext). South Dartmouth (MA).2000

27. Tierney AC, McMonagle J, Shaw DI and Gulseth HL. et al. Effects of dietary fat modification on insulin sensitivity and on other risk factors of the metabolic syndrome--LIPGENE: a European randomized dietary intervention study. Int J Obes (Lond). 2011;35(6):800-9.

\section{SUMMARY}

- Anthocyanin is a kind of functional food factor, Aornia melanocarpa anthocyanin (AMA) with regulating body metabolism and insulin signaling pathway, reduce inflammation, and improve liver tissue morphology and other important functions. It has the potential on develop into health products. 